\title{
POSSIBLY LOST ORCHID TREASURE OF BANGLADESH AND THEIR ENUMERATION WITH CONSERVATION STATUS
}

\author{
Rashid, M. E., M. A. Rahman and M. K. Huda \\ Department of Botany, University of Chittagong, Chittagong 4331, Bangladesh
}

\begin{abstract}
The study aimed at determining the status of occurrence of the orchid treasure of Bangladesh for providing data for Planning National Conservation Strategy and Development of Conservation Management. 54 orchid species are assessed to be presumably lost from the flora of Bangladesh due to environmental degradation and ecosystem depletion. The assessment of their status of occurrence was made based on long term field investigation, collection and identification of orchid taxa; examination and identification of herbarium specimens preserved at CAL, E, K, DACB, DUSH, BFRIH,BCSIRH, HCU; and survey of relevant upto date floristic literature. These species had been recorded from the present Bangladesh territory for more than 50 to 100 years ago, since then no further report of occurrence or collection from elsewhere in Bangladesh is available and could not be located to their recorded localities through field investigations. Of these, 29 species were epiphytic in nature and 25 terrestrial. More than $41 \%$ of these taxa are economically very important for their potential medicinal and ornamental values. Enumeration of these orchid taxa is provided with updated nomenclature, bangla name(s) and short annotation with data on habitats, phenology, potential values, recorded locality, global distribution conservation status and list of specimens available in different herbaria.
\end{abstract}

Key words: Orchid species, lost treasure, Bangladesh, conservation status, assessment.

\section{INTRODUCTION}

The orchid species belonging to the family Orchidaceae are represented mostly in the tropical parts of the world by 880 genera and about 26567 species (Cai et al. 2015) and a few in the arctic regions. In Asia, the family is rich with species diversity, represented by more than 1300 species in India, 579 species in Bhutan (Pearce and Cribb 2002), 450 species in Nepal (Pant et al. 2016) and 187 species in Bangadesh (Rahman et al. 2017).

It was William Roxburgh (1814) who for the first time recorded 12 species of orchids in his Hortus Bengalensis (nom. nud.) from the area now in Bangladesh and later described in his Flora Indica (1932), and many of his collections are still available at BM, K and E. Thereafter Nathalian Wallich (18281849), Hooker (1885-1890; 1890-1894), Kurz (1877), Prain (1903), Heinig (1925), Datta and Mitra (1953) and Sinclair (1956) subsequently made remarkable contributions towards the account of the Orcidaceae with a total of 110 species before independence of Bangladesh in 1971.

While assessing the status of occurrence of these recorded orchid taxa we came across the scenario that 54 recorded species of the family could not be located in their collection localities of Bangladesh during field investigations conducted by the authors themselves and even different works conducted on orchids since 1971 like, Seidenfaden (1978a, b; 1985, 1988), Moyeen (1982), Biswajit (1987), Khan and Halim (1987; 1991), Halim and Khan (1991), Ahmed and Pasha (1993, 1994, 1998, 1998a), Ahmed et al. (1993, 1989, 1989a, 1989b, 1989c, 1991, 1994), Rahman and Huda (1998, 2001), Huda and Rahman (1999), Huda et al. (1999, 2001), Khanam et al. (2001), Huda (2007, 2008), Islam et al. (2016), Rahman et al. (2017).

Moreover there is no report of occurrence of any of these taxa could be found even in differect floristic works of Huq and Khan (1984), Khan et al. (1994), Rahman and Uddin (1997), Uddin et al. (1998), Uddin and Rahman (1999), Khan and Huq (2001), Rashid and Mia (2001), Uddin et al. (2002), Uddin and Hassan (2004), Momen et al. (2006), Islam et al. (2009), Tutul et al. (2009), Uddin and 
Hassan (2010), Arefin et al. (2011), Rahman et al. (2012), Uddin and Hassan (2012), Uddin et al. (2013) and Rahman (2017).

Huda (2008) in his compilation of orchids in the Encyclopedia of Flora and Fauna of Bangladesh cited 179 taxa where 4 previously recorded species were missing. Islam et al. (2016) recently in their list of orchid taxa from greater Sylhet missed 10 species which had been previously recorded in the works of Wallich (1828-1849), Hooker (1885-1890), Barbhuiya and Gogoi (2010).

\section{MATERIAL AND METHODS}

The study has been conducted on the family Orchidaceae of Bangladesh towards the inventory of its species diversity and their status of occurrence. Literature, relevant to the records of orchids of Bangladesh, has been thoroughly surveyed and a list of taxa prepared with their recorded localities in the flora, and these are: Seidenfaden (1978a, b; 1985, 1988), Moyeen (1982), Biswajit (1987), Khan and Halim (1987; 1991), Halim and Khan (1991), Ahmed and Pasha (1993, 1994, 1998, 1998a), Ahmed et al. (1993, 1989, 1989a, 1989b, 1989c, 1991, 1994), Rahman and Huda (1998, 2001), Huda and Rahman (1999), Huda et al. (1999, 2001), Khanam et al. (2001), Huda (2007, 2008), Islam et al. (2016), Rahman et al. (2017).

Upto date floristic literature published with local taxa have also been consulted for occurrence and distribution of orchids in different localities of Bangladesh, and these are: Huq and Khan (1984), Khan et al. (1994), Rahman and Uddin (1997), Uddin et al. (1998), Uddin and Rahman (1999), Khan and Huq (2001), Rashid and Mia (2001), Uddin et al. (2002), Uddin and Hassan (2004), Momen et al. (2006), Islam et al. (2009), Tutul et al. (2009), Uddin and Hassan (2010), Arefin et al. (2011), Rahman et al. (2012), Uddin and Hassan (2012) and Uddin et al. (2013).

The herbaria of local, national and international have been consulted and the specimens of orchids of Bangladesh critically examined, determined and prepared a list with their collection localities in order to locate them in the field and assess their present status of occurrence. The consulted herbaria are: BM, CAL, E, K, DACB, BFRIH (Bangladesh Forest Research Institue Herbarium), BCSIRH (Bangladesh Council for Science and Industrial Research), DUSH (Dakha University Salarkhan Herbaium), HCU (Herbarium of Chittagong University).

Field investigations have been made throughout the flora, especially in the recorded localities of orchid species since 1984 as regular botanical explorations. Identification and collection of orchid taxa have been made following standard taxonomic methods.

An enumeration of these possibly extinct taxa is prepared following Rahman (2013) where each species is cited with current nomenclature, synonym(s), Bangla name(s), habit, flowering and fruiting period, potential values, recorded localities, global distribution and list of herbarium specimens.

\section{RESULTS AND DISCUSSION}

The study revealed that $54(\%)$ of the 187 recorded orchid taxa of Bangladesh are thought to be possibly lost from the flora according to the definition of extinct category (IUCN 2001) since these have no report of occurrence or could not be located in the wild for more than 50 to 100 years. This result coincide the report of lost asclepiad taxa (28.36\%) by Rahman (2013). The data presented in the enumeration revealed that two species have no further report of collection from elsewhere in Bangladesh since Roxburgh (1832), six species since Wallich (1828-49), 29 species since Hooker (1885-1890), one species since Prain (1903), eight species since Heinig (1925) and eight species since Sinclair (1956).

Tweenty five species recorded from greater Sylhet and thirteen species from Chittagong/Hill Tracts area until Hooker (1885-1890) have no further report in any later works. Dendrobium transparens Wall. ex Lindl., recorded from Sylhet by Barbhuiya and Gogoi (2010) based on collection of 1943, have no 
report of second collection for more than 73 years. Rahman and Jahan (2016) recently reported about 9\% of medicinal species of the Fabceae possibly lost from Bangladesh. The study also revealed that 29 (\%) species were epiphytic and $25(\%)$ terrestrial, and $22(41 \%)$ of these were medicinal and $21(39 \%)$ ornamental.

Orchids in the wild mostlly found to grow as epiphytic on the trunk of old trees, like rain tree, dipterocarps, sal, jarul, banyan, mango etc. mostly in the rain forests of Chittagong, Cox's Bazar, Hill Tracts districts, Tangail and greater Sylhet. There are many deep forests in Hill Tracts districts most of which are inaccesible due to tribal/ political conflicts. It has been deficult to collect and locate many rare orchids from those forested areas. Moreover many old forests in greater Sylhet district existed before 1947, either fully or partially destroyed and many recorded rare orchids of both epiphytic and ground either disappeared or could not be located in their recorded habitats which are very much degraded now. Some of these taxa, thought to be extinct, could probably still be existed in remote/deep forest habitats. The family has not yet been inventoried for the Flora of Bangladesh and identification, collection and preservation of orchid taxa were not adequate and seemed to be insufficient works and publications have been made on the family. Due attention to the assessment and conservation management in the Orchids of Bangladesh has not been paid in the National Conservation Strtegy. More intensive investigations to the very remote areas for collection and identification of orchids are required which may provide the rediscovery of some of these recorded taxa, thought to be lost. For instance, at least 7 species of orchids could be relocated by recent investigations, e.g. Coelogyne cristata Lindl. Hooker (1885-1890), Oberonia gammiei King and Pantl. Prain (1903) and Eria bractescens Lindl. Heinig (1925).

The loss of 54 species orchids from the flora of Bangladesh by 100 years warns that the family Orchidaceae is in most vulnerable state and need immediate and proper conservation measures before their disappearance from the wild for sustainability of the environment.

However, the study provided the status of occurrence and rate of extinction of orchid taxa in the wild, and data of recorded/collected localities on which basis further attempts for their rediscovery and conservation management could be taken (in-situ/ex-situ) following National Conservation Strategy of Bangladesh.

\section{Enumeration of possibly lost orchid taxa of Bangladesh}

1. Acampe cephalotes Lindl., Fol. Orch. 4: 3 (1853).

Gastrochilus cephalotes (Lindl.) Kuntze (1891); Saccolabium cephalotes (Lindl.) Hook.f. (1890). Bangla name: Not known. Habitat: Epiphytic. Fl.-Fr.: Not on record. Potential value: Ornamental.

Recorded locality: Sylhet (lnc). No report of collection Hook.f. (1890).

Global distribution: Bnagladesh, Cambodia, Chins, India, Laos, Myanmar, Nepal, Taiwan and Vietnam.

Herbarium specimens: Sylhet: 19.11.1947, J. D. Hooker and T. Thomson 197 (K). No herbarium specimens available in DACB, DUSH, HCU, BFRIH and BCSIRH.

2. Acanthephippium sylhetense Lindl., Gen. Sp. Orch. Pl.: 177 (1831).

Acanthephippium ringiflorum Griff. (1851). Bangla name: Not known. Habitat: Terrestrial. Fl.-Fr.: April

- May (Bose et al. 1999). Potential Value: Medicinal (http://www.ecoindia.com/flora/s/orchid).

Recorded locality: Sylhet (lnc). No report of collection Hook.f. (1890).

Golbal distribution: Bangladesh, China, NE India, Japan, Laos, Malaysia, Myanmar and Thailand.

Herbarium specimens: No herbarium specimens available in DACB, DUSH, HCU, BFRIH and BCSIRH.

3. Anoectochilus setaceus Blume, Bijdr.: 412 (1825).

Anaectochilus roxburghii (Wall.) Lindl. (1839); A. setaceus Lindl. (1837) nom. illeg.; Chrysobaphus roxburghii Wall. (1826); Orchis picta Reinw. ex Lindl. (1857). Bangla name: Not known. Habitat: 
Terrestrial. Fl.-Fr.: April - June (Peaece and Cribb 2002). Potential Value: Medicinal (Ratna et al. 2014).

Recorded locality: Sylhet (lnc). No report of collection Hook.f. (1890).

Golbal distribution: Bangladesh, Bhutan, China, India, Japan, Laos, Nepal, Thailand and Vietnam. Herbarium specimens: No herbarium specimens available in DACB, DUSH, HCU, BFRIH and BCSIRH.

4. Ascocentrum ampullaceum (Roxb.) Schltr. in Repert. Spec. Nov. Regni Veg. Beih. 1: 975 (1913). Aerides ampullacea Roxb. (1832); Oeceoclades ampullacea (Roxb.) Lindl. ex Voigt (1845); Gastrochilus ampullaceus (Roxb.) Kuntze (1891); Saccolabium ampullaceum (Roxb.) Lindl. (1832). Bangla name: Not known. Habitat: Epiphytic. Fl.-Fr.: March-May (Yonzone et al. 2012). Potential Value: Not known.

Recorded locality: Sylhet (lnc). No report of collection Hook.f. (1890).

Golbal distribution: Bangladesh, Bhutan, China, India (including Andaman Islands), Laos, Myanmar, Nepal, and Thailand.

Herbarium specimens: No herbarium specimens available in DACB, DUSH, HCU, BFRIH and BCSIRH.

5. Calanthe densiflora Lindl., Gen. Sp. Orchid. Pl.: 250 (1833).

Alismorkis densiflora (Lindl.) Kuntze (1891); Calanthe kazuoi Yamamoto (1930). Bangla name: Not known. Habitat: Terrestrial. Fl.-Fr.: August - October (Lang et al.1999). Potential Value: Not known.

Recorded locality: Sylhet (lnc). No report of collection Wallich (1824).

Golbal distribution: Bangladesh, Bhutan, China, India, Japan, Nepal and Vietnam.

Herbarium specimens: Sylhet: F. De Silva, Wall. Cat. 7344 (K). No herbarium specimens are available in DACB, DUSH, HCU, BFRIH and BCSIRH.

6. Calanthe puberula Lindl., Gen. Sp. Orchid. Pl.: 252 (1833).

Alismorkis puberula (Lindl.) Kuntze (1891); Calanthe amoena W. W. Smith (1921); C. lepida W. W. Smith (1921). Bangla name: Not known. Habitat: Terrestrial. Fl.-Fr.: October - December (Peaece and Cribb 2002). Potential Value: Medicinal (Pant 2013).

Recorded locality: Sylhet (lnc). No report of collection Lindley (1830-1840).

Golbal distribution: Bangladesh, Bhutan, China, India, Japan, Myanmar, Nepal, Taiwan and Vietnam.

Herbarium specimens: Sylhet: F. D. Silva, Wall. Cat. no 7342 (K). But no herbarium specimens are available in DACB, DUSH, HCU, BFRIH and BCSIRH.

7. Cleisostoma subulatum Blume, Bijdr.: 363 (1825).

Cleisostoma dealbatum Lindl. (1843); Sarcanthus secundus Griff. (1851); S. oxyphyllus Wall. ex Reichb.f. (1855); S. subulatus (Blume) Reichb.f. (1857); S. secundus Griff. (1851). Bangla name: Not known. Habitat: Epiphytic. Fl.: August - October (Internet). Potential Value: Not known.

Recorded locality: Chittagong (lnc) (Prain 1903), CHT (lnc) (Heinig 1925), Sundribans (Prain 1903), Sylhet $(\ln c)$ (Hook.f. 1890). No report of collection Heinig (1925).

Golbal distribution: Bangladesh, Bhutan, India, eastern Himalayas, Myanmar, Thailand, Cambodia, Vietnam, Malaysian peninsula, Sumatra, Java, Lesser Sunda Islands, Borneo, the Moluccas and the Philippines.

Herbarium specimens: No herbarium specimens available in DACB, DUSH, HCU, BFRIH and BCSIRH. 
8. Coelogyne punctulata Lindl., Coll. Bot. ad t. 33 (1821).

Coelogyne brevifolia Lindl.; C. nitida (Roxb. ) Hook.f. (1890), C. ocellata Lindley; Cymbidium nitidum Roxb. (1832), not Wallich ex D. Don (1825); Pleione goweri (Reich.) Kuntze (1891); P. nitida Kuntze (1891). Bangla name: Not known. Habitat: Epiphytic. Fl.-Fr.: March - April (Peaece and Cribb 2002). Potential Value: Medicinal (Deb et al. 2009).

Recorded locality: Sylhet $(\operatorname{lnc})$. No report of collection after Roxburgh (1832) by its synonym Cymbidium nitidum Roxb.

Golbal distribution: Bangladesh, Bhutan, China, NE India, Myanmar and Nepal.

Herbarium specimens: No herbarium specimens available in DACB, DUSH, HCU, BFRIH and BCSIRH.

9. Crepidium biauritum (Lindl.) Szlach., Fragm. Florist. Geobot. Suppl. 3: 124 (1995).

Crepidium andamanicum (King and Pantl.) Marg. and Szlach. (2001); Malaxis andamanica (King and Pantl.) N. P. Balakr and V. Rao (1981); M. brevicaulis (Schltr.) S. Y. Hu (1974); M. biaurita Kuntze (1891); Microstylis andamanica King and Pantl. (1897); M. biaurita Lindl. (1830); M. brevicaulis Schltr. (1919); M. thorelii Finet (1907). Bangla name: Not known. Habitat: Terrestrial. Fl.-Fr.: July October (Hook.f. 1890). Potential Value: Medicinal (San et al. 2015).

Recorded locality: Sylhet (Pandua). No report of collection Wallich (1832).

Golbal distribution: Andaman Islands, Bangladesh, China, India, Laos, Myanmar, Nepal, Thailand.

Herbarium specimens: Sylhet: Pandua, 1832, Wallich, Wall. Cat. 1941 (K). But no herbarium specimens are available in DACB, DUSH, HCU, BFRIH and BCSIRH.

10. Crepidium calophyllum (Rchb.f.) Szlach., Fragm. Florist. Geobot. Suppl. 3: 125 (1995).

Epidendrum variegatum König (1791); Liparis elegantissima Ridl. (1888); Malaxis calophylla (Reichb. f.) Kuntze (1891); M. scottii (Hook.f.) Kuntze (1891); Microstylis calophylla Reichb. f. (1879); M. scottii Hook.f. (1890); M. wallichii var. brachycheila Hook.f. (1890). Bangla name: Not known. Habitat: Terrestrial. Fl.-Fr.: July (Peaece and Cribb 2002). Potential Value: Horticultural.

Recorded locality: Dhaka (lnc). No report of collection after Hook.f. (1890).

Golbal distribution: Bangladesh, Borneo, Cambodia, China, India (Sikkim), Indonesia, Malaysia, Myanmar, Thailand and Vietnam.

Herbarium specimens: No herbarium specimens available in DACB, DUSH, HCU, BFRIH and BCSIRH.

11. Dendrobium angulatum Lindl. Gen. Sp. Orchid. Pl. 88 (1830).

Callista angulata Kranzl (1891); Dendrobium inconcinnum Ridl. (1896); D. podagraria Hook.f. (1890) nom. illeg. Bangla name: Not known. Habitat: Epiphytic. Fl.-Fr.: April - May (Gogoi et al. 2014). Potential Value: Horticultural plant.

Recorded locality: Chittagong (lnc; Prain 1903) and CHT (lnc; Heinig 1925). No report of collection Heinig (1925).

Golbal distribution: Bangladesh, India, Myanmar, southwestern Thailand and Vietnam.

Herbarium specimens: Bangladesh: 1821, De Silva 2001/2 (K). But no herbarium specimens are available in DACB, DUSH, HCU, BFRIH and BCSIRH.

12. Dendrobium blumei Lindl., Sp. Orchid. Pl. 88 (1830).

Aporum blumei (Lindl.) Rauschert (1983); Callista boothii (Teijsm. and Binn.) Kuntze (1891); C. tuberifera (Hook.f.) Kuntze (1891); Dendrobium tuberiferum Hook.f. (1890); Onychium fimbriatum Blume (1825). Bangla name: Not known. Habitat: Epiphytic. Fl.-Fr.: Late winter and early spring (IOSPE PHOTOS). Potential Value: Not known. 
Recorded locality: Chittagong (lnc). No report of collection Hook.f. (1890).

Golbal distribution: Bangladesh, Borneo, Java, Malaysia and Thailand.

Herbarium specimens: No herbarium specimens are available in DACB, DUSH, HCU, BFRIH and

BCSIRH.

13. Dendrobium chryseum Rolfe, Gard. Chron. Ser. 3, 3: 233 (1888).

Callista aurantiaca Kuntze (1891); Dendrobium aurantiacum Reichb.f. (1887); D. clavatum Wall. ex Lindl. (1852); D. flaviflorum Hayata (1911); D. rivesii Gagnep. (1930); D. tibeticum Schltr. (1921). Bangla name: Not known. Habitat: Epiphytic. Fl.-Fr.: June (Peaece and Cribb 2002). Potential Value: Not known.

Recorded locality: Sylhet (lnc). No report of collection Hook.f. (1890).

Golbal distribution: Bangladesh, Bhutan, China, eastern Himalayas, India, Laos, Myanmar, Nepal, Taiwan, Thailand, Vietnam and Western Himalayas.

Herbarium specimens: No herbarium specimens are available in DACB, DUSH, HCU, BFRIH and BCSIRH.

14. Dendrobium crepidatum Lindl. and Paxton, Paxt. Fl. Gard. 1: 63 (1850).

Callista crepidata (Lindl. and Paxton) Kuntze (1891); Dendrobium lawanum Lindl. (1859); Dendrochilum roseum Dalz. (1852). Bangla name: Not known. Habitat: Epiphytic. Fl.-Fr.: April - May (Abraham and Vatsala 1981). Potential Value: Medicinal (Nongdam 2014; Pant 2013).

Recorded locality: Cox's Bazar (Below Pagoda on hill side). No report of collection Sinclair (1956). Golbal distribution: Bangladesh, Bhutan, China, India, Laos, Myanmar, Nepal, Thailand and Vietnam.

Herbarium specimens: Cox's Bazar: Below Pagoda on hill side, 01.03.1945, Sinclair s.n. (K). No herbarium specimens are available in DACB, DUSH, HCU, BFRIH and BCSIRH.

15. Dendrobium lituiflorum Lindl., Gard. Chron.: 372 (1856).

Callista lituiflora (Lindl.) Kuntze (1891); Dendrobium hanburyanum Reichb.f (1856). Bangla name: Not known. Habitat: Epiphytic. Fl.-Fr.: February - April (Huda 2000). Potential Value: Horticultural.

Recorded locality: CHT (lnc; Heinig 1925), Cox's Bazar (lnc; Sinclair 1956). No report of collection from Cox's Bazar Sinclair (1956).

Golbal distribution: Bangladesh, China, India, Laos, Myanmar, Thailand and Vietnam.

Herbarium specimens: No herbarium specimens are available in DACB, DUSH, HCU, BFRIH and BCSIRH.

16. Dendrobium longicornu Lindl., Gen. Sp. Orchid. Pl. 80 (1830).

Callista longicornis (Lindl.) Kuntze (1891); Dendrobium bulleyi Rolfe (1913); D. flexuosum Griff. (1851); D. hirsutum Griff. (1851); Froscula hispida Raf. (1838). Bangla name: Not known. Habitat: Epiphytic. Fl.-Fr.: August - December (Huda 2008). Potential Value: Medicinal (Pant 2013).

Recorded locality: Sylhet (lnc). No report of collection de Silva (1821).

Golbal distribution: Bangladesh, Bhutan, China, NE India, Myanmar, Nepal, and Vietnam.

Herbarium specimens: Sylhet: 1821, F. De Silva 1997(2), (K). But no herbarium specimens available in DACB, DUSH, HCU, BFRIH and BCSIRH.

17. Dendrobium moschatum (Buch.-Ham.) Sw. in Schltr., Neues J. Bot. 1: 94 (1805).

Callista calceola (Carey ex Hook.) Kuntze (1891); C. moschata Kuntze (1891); Cymbidium moschatum Willd. (1805); Dendrobium calceolaria Carey ex Hook. (1825); D. cupreum Herb. ex Lindl. (1835); D. moschatum Wall. ex D. Don (1825); D. calceolaria Carey ex Hook. (1825); Epidendrum moschatum 
Buch.-Ham. (1800). Bangla name: Not known. Habitat: Epiphytic. Fl.-Fr.: May - July (Huda 2000). Potential Value: Medicinal (Pant 2013).

Recorded locality: Chittagong (lnc; Prain 1903), Cox's Bazar (near Buddhist Monastery; Sinclair 1956), CHT (lnc; Heinig 1925), Sylhet (near Forest School). No collection Sarkar (1956).

Golbal distribution: Bangladesh, Bhutan, China, India, Laos, Myanmar, Nepal, Thailand and Vietnam. Conservation measures proposed: Further search should be made to relocate this species, if exists, then in-situ or ex-situ or both conservation measures to be taken as appropriate.

Herbarium specimens: Cox's Bazar: near Buddhist Monastery, 09.05.1945, Sinclair s.n. (K). Sylhet: near Forest School, 13.03.1956, Sarkar s.n. (DUSH) and no herbarium specimens available in DACB, HCU, BFRIH and BCSIRH.

18. Dendrobium ochreatum Lindl. in Edwards's Bot. Reg. 21 (Misc.): t. 1756 (1835).

Callista ochreata (Lindl.) Kuntze (1891); Dendrobium cambridgeanum Paxton (1839). Bangla name: Not known. Habitat: Epiphytic. Fl.-Fr.: February - April (Huda 2000). Potential Value: Medicinal (San et al. 2015).

Recorded locality: Chittagong (lnc; Hook.f. 1890, Prain 1903) and CHT (lnc; Heinig 1903). No report of collection Heinig (1925).

Golbal distribution: Bangladesh, India, Myanmar, Thailand and Vietnam.

Herbarium specimens: Chittagong: a. 1828, Bruce, Wall. Cat. 7410 (K). But no herbarium specimens available in DACB, DUSH, HCU, BFRIH and BCSIRH.

19. Dendrobium pachyphyllum (Kuntze) Bakh.f. in Blumea 12: 69 (1963).

Bolbodium pumilum (Kuntze) Brieger (1981); B. pusillum (Blume) Rausch. (1983); Callista pachyphylla Kuntze (1891); C. pusilla (Blume) Kuntze (1891); C. pumila Kuntze (1891); Desmotrichum pusillum Blume (1825); Dendrobium pusillum (Blume) Lindl. (1830) nom. illeg.; D. pumilum Roxb. (1832) nom. illeg; D. perpusillum Balakar. (1970). Bangla name: Not known. Habitat: Epiphytic. Fl.-Fr.: July August (Bhattacharjee and Dutta 2010). Potential Value: Medicinal (Kirtikar and Basu 1981).

Recorded locality: Chittagong (lnc), CHT (lnc), Cox's Bazar $(\ln c)$. No report of collection from Cox's Bazar Ompdale and Ahmed (1945).

Golbal distribution: Bangladesh, Borneo, India, Java, Malaysia, Myanmar, Sumatra, Thailand and Vietnam.

Herbarium specimens: Cox's Bazar: 06.07.1945, Ompdale and Ahmed s.n. (DUSH) and no herbarium specimens available in DACB, HCU, BFRIH and BCSIRH.

20. Dendrobium palpebrae Lindl., J. Hort. Soc. 5 : 33 (1850).

Callista palpebrae (Lindl.) Kuntze (1891); C. palpebrae (Lindl.) Brieger (1981); Dendrobium farmeri var. album Regel (1868). Bangla name: Not known. Habitat: Epiphytic. Fl.-Fr.: April - May (Bhattacharjee and Dutta 2010). Potential Value: Horticultural plant.

Recorded locality: Chittagong (lnc; Prain 1903) and CHT (lnc; Heinig 1925). No report of collection Heinig (1925).

Golbal distribution: Bangladesh, India, Laos, Myanmar Thailand and Vietnam.

Herbarium specimens: No herbarium specimens available in DACB, DUSH, HCU, BFRIH and BCSIRH.

21. Dendrobium pulchellum Roxb. ex Lindl., Gen. Sp. Orchid. Pl.: 82 (1830).

Callista pulchella (Roxb. ex Lindl.) Kuntze (1891); Dendrobium brevifolium Lindl. (1858); D. dalhousieanum Wall. (1844); D. moschatum Griff. (1851). Bangla name: Not known. Habitat: Epiphytic. Fl.-Fr.: February - April (Hook.f. 1890). Potential Value: Medicinal (San et al. 2015). 
Recorded locality: Sylhet (lnc). No report of collection Roxburgh (1832).

Golbal distribution: Bangladesh, eastern Himalayas, India, Laos, Malaysia, Myanmar, Nepal, Thailand, Vietnam and Yunnan China.

Herbarium specimens: No herbarium specimens are available in DACB, DUSH, HCU, BFRIH and BCSIRH.

22. Dendrobium ruckeri Lindl. in Edwards's Bot. Reg. 29 (Misc.): 25 (1843).

Callista ramosa (Lindl.) Kuntze (1891); C. ruckeri (Lindl.) Kuntze (1891); Dendrobium ramosum Lindl. (1830) nom. illeg. Bangla name: Not known. Habitat: Epiphytic. Fl.-Fr.: Not on record. Potential Value: Horticultural Plant.

Recorded locality: Sylhet (Pandua). No report of collection Hook.f. (1890).

Golbal distribution: Bangladesh, eastern Himalayas, India, Laos and Myanmar.

Herbarium specimens: Sylhet: Pandua, a. 1832, Wallich, Wall. Cat. 2003 (K). But no herbarium specimens available in DACB, DUSH, HCU, BFRIH and BCSIRH.

23. Dendrobium transparens Wall. ex Lindl., Gen. Sp. Orchid. Pl.: 79 (1830).

Callista transparens (Wall.) Kuntze (1891); Dendrobium henshalli Reichb.f. (1855). Bangla name: Not known. Habitat: Epiphytic. Fl.-Fr.: May (Huda 2000). Potential Value: Medicinal (Pant 2013).

Recorded locality: Chittagong (lnc; Prain 1903), HT (lnc; Heinig 1903), Sylhet (lnc). No report of collection from Sylhet Deka (1943).

Golbal distribution: Bangladesh, Bhutan, India, Myanmar and Nepal.

Herbarium specimens: Sylhet: 30.04.1943, G.K. Deka 22189 (ASSAM) but no herbarium specimens are available in DACB, DUSH, HCU, BFRIH and BCSIRH.

24. Eria paniculata Lindl. , Pl. Asiat. Rar. 1: 32 (1830).

Callista floribunda (D. Don) Kuntze (1891); Dendrobium floribundum D. Don (1825); Mycaranthes floribunda (D. Don) S.C. Chen \& J.J. Wood (2009); Pinalia paniculata (Lindl.) Kuntze (1891). Bangla name: Not known. Habitat: Terrestrial or Epiphytic. Fl.-Fr.: January - May (Peaece and Cribb 2002). Potential Value: Not known.

Recorded locality: Sylhet (lnc). No report of collection Wallich (1824).

Golbal distribution: Bangladesh, Cambodia, Chinese Himalayas, Laos, the eastern Himalayas, Myanmar, Nepal, Thailand and Vietnam.

Herbarium specimens: Sylhet: 1824, Silva, Wall. Cat. no. 1971 (K). But no herbarium specimens are available in DACB, DUSH, HCU, BFRIH and BCSIRH.

25. Erythrodes blumei (Lindl.) Schltr. in Fl. Schutzgeb. Sudsee Nachtr.: 87 (1905).

Erythrodes humilis (Blume) J. J Smith (1907); Microchilus blumei (Lindl.) D. Dietr (1852); Physurus humilis Blume (1859); P. blumei Lindl. (1840); P. chinensis Rolfe (1896). Bangla name: Not known. Habitat: Terrestrial. Fl.-Fr.: January - March (Jayaweera 1981). Potential Value: Horticultural plant.

Recorded locality: Sylhet (lnc; Lindley 1830-1840; Hook.f. 1890). No report of collection after Hook.f. (1890) by its synonym Physurus blumei Lindl.

Golbal distribution: Bangladesh, China, India, Indonesia, Java, Malaysia, Myanmar, Sumatra, Thailand and Vietnam.

Herbarium specimens: Sylhet: 1832, F. De Silva, Wall. Cat. 7397 (K, K-W). But no herbarium specimens are available in DACB, DUSH, HCU, BFRIH and BCSIRH. 
26. Eulophia bracteosa Lindl., Gen. Sp. Orch. Pl.: 180 (1833).

Eulophia grandiflora Lindl. (1833); Geodorum bracteatum Voigt (1845); Graphorkis bracteosa (Lindl.) Kuntze (1891). Bangla name: Not known. Habitat: Terrestrial. Fl.-Fr.: August (Hook.f. 1890). Potential Value: Horticultural plant.

Recorded locality: Chittagong (lnc; Hook.f. 1890, Prain 1903); HT (Heinig 1925). No report of collection Heinig (1925).

Golbal distribution: Bangladesh, China, India and Myanmar.

Herbarium specimens: Chittagong: no date, Bruce Wall. Cat. no. 7366 (K). But no herbarium specimens are available in DACB, DUSH, HCU, BFRIH and BCSIRH.

27. Eulophia dabia (D. Don) Hochr., Bull. New York Bot. Gard. 6: 270 (1910).

Bletia dabia D. Don (1825); Eulophia campestris Wall. ex Lindl. (1833); Eulophia ramentacea (Roxb.) Lindl. (18330; E. rupestris Wall. ex Lindl. (1833); Geodorum ramentaceum (Roxb.) Voigt (1845); Graphorkis campestris (Wall. ex Lindl.) Kuntze (1891); G. dabia (D. Don) Kuntze (1891); G. rupestris (Wall. ex Lindl.) Kuntze (1891); Limodorum dabia (D. Don) Buch.-Ham. ex D. Don (1825); L. ramentaceum Roxb. (1832). Bangla name: Salab Misri, Pranada. Habitat: Terrestrial c. Fl.-Fr.: March (Ahmed and Pasha 1998). Potential Value: Medicinal (Pant 2013).

Recorded locality: Chittagong (lnc). No report of collection after Heinig (1925) by its synonym Eulophia campestris Wall. ex Lindl.

Golbal distribution: Afghanistan, Bangladesh, Bhutan, China, India, Kashmir, Nepal, Pakistan, Tajikistan, Turkmenistan and Uzbekistan.

Herbarium specimens: No herbarium specimens available in DACB, DUSH, HCU, BFRIH and BCSIRH.

28. Eulophia herbacea Lindl., Gen. Sp. Orch. Pl.: 182 (1833).

Eulophia vera Royle (1839); E. albiflora Edgew. ex Lindl. (1859); E. brachypetala Lindl. (1859); E. lachnocheila Hook.f. (1890); Geodorum bicolor (Roxb.) Voigt (1845); Limodorum bicolor Roxb. (1832). Bangla name: Not known. Habitat: Terrestrial. Fl.-Fr.: May -July (Ahmed and Pasha 1998). Potential Value: Medicinal (Patil and Mahajan 2013). Bulb edible. A nutritious starch. (Ken 2014).

Recorded locality: Rangpur (lnc). No report of collection Hook.f. (1890). Golbal distribution: Bangladesh, China, India, Laos, Nepal and Thailand.

Herbarium specimens: No herbarium specimens are available in DACB, DUSH, HCU, BFRIH and BCSIRH.

29. Gastrochilus calceolaris (Buch.-Ham. ex Smith) D. Don, Prodr. Fl. Nepal.: 32 (1825).

Aerides calceolaris Buch.-Ham. ex Smith (1818); A. leopardorum Wall. ex Hook.f. (1890); Epidendrum calceolare (Buch.-Ham. ex Smith) D. Don (1825); Sarcochilus nepalensis Spreng. (1826) nom. illeg.; Saccolabium calceolare (Buch.-Ham. ex Smith) Lindl. (1833). Bangla name: Not known. Habitat: Epiphytic. Fl.-Fr.: March - May (Peaece and Cribb 2002). Potential Value: Medicinal (Oudhia 2012).

Recorded locality: Sylhet (lnc). No report of collection Hook.f. (1890).

Golbal distribution: Bangladesh, Bhutan, China, India, Malaysia (Peninsular), Myanmar, Nepal, Thailand and Vietnam.

Herbarium specimens: No herbarium specimens are available in DACB, DUSH, HCU, BFRIH and BCSIRH.

30. Geodorum recurvum (Roxb.) Alston in Handb. Fl. Ceylon 6 (Suppl.): 276 (1931).

Geodorum dilatatum R. Br. (1813); Limodorum recurvum Roxb. (1795). Bangla name: Not known. Habitat: Terrestrial. Fl.-Fr.: April -Junnuary (Lang et al. 1999). Potential Value: Medicinal (Hossain 2011). 
Recorded locality: Sylhet (lnc). No report of collection Hook.f. (1890).

Golbal distribution: Bangladesh, China, Cambodia, India, Myanmar, Thailand and Vietnam.

Herbarium specimens: No herbarium specimens are available in DACB, DUSH, HCU, BFRIH and BCSIRH.

31. Habenaria digitata Lindl., Gen. Sp. Orch. Pl.: 307 (1833).

Bonatea punduana Lindl. ex Wall (1828-49) ined.; B. herbacea Wall. ex Lindl. (1835); B. benghalensis Griff. (1844); Habenaria laciniata Dalz. (1850); H. trinervia Wight (1851); H. travancorica Hook.f. (1890). Bangla name: Not known. Habitat: Terrestrial. Fl.-Fr.: August -November (Joseph 1987). Potential Value: Medicinal (Oudhia 2012).

Recorded locality: Sylhet (lnc; Hook.f. 1890); East Bengal (lnc; Prain 1903). No report of collection after Prain (1903) from East Bengal and also No report of collection from Sylhet Hook.f. (1890).

Golbal distribution: Bangladesh, Cambodia, China, eastern Himalayas, Laos, Myanmar, Nepal, Pakistan and western Himalayas.

Herbarium specimens: No herbarium specimens are available in DACB, DUSH, HCU, BFRIH and BCSIRH.

32. Habenaria diphylla (Nimmo) Dalz. in Hook., J. Bot. Kew Gard. Misc. 2: 262 (1850).

Habenaria jardoniana Wight (1851); H. sutteri Reichb.f. (1852); Liparis diphyllos Nimmo (1839).

Bangla name: Not known. Habitat: Terrestrial. Fl.-Fr.: June -September (Joseph 1987). Potential Value: Horticultural plant.

Recorded locality: Dhaka (lnc). No report of collection after Hook.f. (1890).

Golbal distribution: Bangladesh, Bhutan, China, eastern Himalayas, India, Myanmar, Nepal, Thailand and the Philippines.

Herbarium specimens: No herbarium specimens are available in DACB, DUSH, HCU, BFRIH and BCSIRH.

33. Habenaria gracilis Coleb., Exot. Fl.: t. 135 (1824).

Bonatea gracilis Lindl. (1835). Bangla name: Not known. Habitat: Terrestrial. Fl.-Fr.: Not on record. Potential Value: Not known.

Recorded locality: Sylhet (lnc). No report of collection after Hook.f. (1890).

Golbal distribution: Bangladesh and Brazil.

Herbarium specimens: No herbarium specimens are available in DACB, DUSH, HCU, BFRIH and BCSIRH.

34. Liparis gamblei Hook.f., Icon. Pl. Ser. 3(19): t. 1812 (1889).

Leptorkis gamblei (Hook.f.) Kuntze (1891). Bangla name: Not known. Habitat: Terrestrial. Fl.-Fr.: June - July (Peaece and Cribb 2002). Potential Value: Not known.

Recorded locality: CHT (lnc). No report of collection is available in CAL Lister (1876).

Golbal distribution: Bangladesh, Bhutan, NE India and Vietnam.

Herbarium specimens: No herbarium specimens available in DACB, DUSH, HCU, BFRIH and BCSIRH.

35. Luisia volucris Lindl., Fol. Orch. 4: 1 (1853).

Not available. Bangla name: Not known. Habitat: Epiphytic. Fl.-Fr.: March -April (Huda 2000).

Potential Value: Horticultural.

Recorded locality: Chittagong (lnc; Hook.f. 1890; Prain 1903), Sylhet (lnc; Hook.f. 1890); HT (Inc; Heinig 1925). No report of collection Heinig (1925).

Golbal distribution: Bangladesh, Borneo, India and the eastern Himalayas.

Herbarium specimens: No herbarium specimens are available in DACB, DUSH, HCU, BFRIH and BCSIRH. 
36. Oberonia mannii Hook.f., Ic. Pl. 21: t. 2003 (1890).

Not available. Bangla name: Not known. Habitat: Epiphytic. Fl.-Fr.: March - June (Lang et al. 1999).

Potential Value: Not known.

Recorded locality: Sylhet (lnc). No report of collection Hook.f. (1890).

Golbal distribution: Bangladesh, China and India.

Herbarium specimens: No herbarium specimens available in DACB, DUSH, HCU, BFRIH and BCSIRH.

37. Pachystoma pubescens Blume, Bijdr. 376, t. 29 (1825).

Apaturia chinensis Lindl. (1831); A. lindleyana Wight (1851); A. montana Lindl. (1831); A. senilis Lindl. (1831); Epipactis scariosa Buch.-Ham. (1831); Neottia pantanis Korth. ex Blume (1856); Pachychilus chinensis (Lindl.) Blume (1856); P. montanus (Lindl.) Blume (1856); P. pantanus Blume (1856); Pachystoma senile (Lindl.) Reichb.f. (1858); P. senile (Lindl.) Trimen (1885) nom. illeg.; Pachystoma fortunei Reichb.f. (1852); P. gracile Schltr. (1906); P. speciosum (Blume) Reichb.f. (18580; P. wightii Reichb.f. (1862). Bangla name: Not known. Habitat: Terrestrial. Fl.-Fr.: February May (Pearce and Cribb 2002). Potential Value: Not known.

Recorded locality: Cox's Bazar (Behind Kelatuli). No report of collection after Sinclair (1945) by its synonym Pachystoma senile (Lindl.) Reichb.f.

Golbal distribution: Australia, Bangladesh, Bhutan, Cambodia, China, India, Indonesia, Laos, Malaysia, Myanmar, Nepal, New Guinea, Philippines and Vietnam.

Herbarium specimens: Cox's Bazar: Behind Kelatuli, 24.02.1945, Sinclair s.n. (K). No herbarium specimens are available in DACB, DUSH, HCU, BFRIH and BCSIRH.

38. Paphiopedilum insigne (Wall. ex Lindl.) Pfitz., Jahrb. Wiss. Bot. 19: 159 (1888).

Cypripedium insigne Wall. ex Lindl. 1824; Paphiopedilum insigne var. sylhetense (Desbois) Pfitz. (1903); Paphiopedilum insigne var. wallacei (Desbois) Pfitzer (1903); Paphiopedilum macfarlanei F.G . Mey (1934). Bangla name: Not known. Habitat: Terrestrial. Fl.-Fr.: October-March (Hook.f. 1890). Potential Value: Horticultural.

Recorded locality: Sylhet (lnc). No report of collection Wallich (1824).

Golbal distribution: Bangladesh, China, India and Nepal.

Herbarium specimens: Sylhet: 1824, F. De Silva, Wall. Cat. 7022 (K). But no herbarium specimens are available in DACB, DUSH, HCU, BFRIH and BCSIRH.

39. Paphiopedilum venustum (Wall. ex Sims) Pfitzer., Jahrb. Wiss. Bot. 19: 165 (1888).

Cypripedium venustum Wall. ex Sims (1820); C. pardinum Reichb.f. (1869) Cordula venusta (Wall. ex Sims) Rolfe (19120; Stimegas venustum (Wall. ex Sims) Raf. (1838). Bangla name: Not known. Habitat: Terrestrial. Fl.-Fr.: Marchy - May (Pearce and Cribb 2002). Potential Value: Not known.

Recorded locality: Sylhet (lnc). No report of collection Hook.f. (1890).

Golbal distribution: Bangladesh, Bhutan, China, India and Nepal.

Herbarium specimens: Sylhet: Wallich and C.; Wall. Cat. 7023 (K-W). But no herbarium specimens are available in DACB, DUSH, HCU, BFRIH and BCSIRH.

40. Pecteilis triflora (D. Don) Tang \& Wang, Acta Phytotax. Sin. 1: 62 (1951).

Habenaria triflora D. Don (1825); H. uniflora Buch.-Ham. ex D. Don (1825); Platanthera candida Lindl. (1835) nom. illeg.; P. uniflora (D. Don) Lindl. (1835); Orchis uniflora Buch.-Ham. ex D. Don (1825). Bangla name: Not known. Habitat: Terrestrial. Fl.-Fr.: August (Huda 2008). Potential Value: Horticultural.

Recorded locality: Mymensingh (lnc). No report of collection Hook.f. (1890).

Golbal distribution: Bangladesh, India and Nepal. 
Herbarium specimens: No herbarium specimens are available in DACB, DUSH, HCU, BFRIH and BCSIRH.

41. Phalaenopsis deliciosa Reichb. f., Bonplandia 2: 93 (1854).

Aerides latifolia Thw. (1864) nom. illeg.; Doritis latifolia Trimen (1885); D. wightii (Reichb.f.) Benth. and Hook.f. (1883); Phalaenopsis wightii Reichb. f. (1862); Kingidium deliciosum (Reichb. f.) Sweet (1970); Kingiella decumbens (Griff.) Rolfe (1917). Bangla name: Not known. Habitat: Epiphytic. Fl.Fr.: Throughout the year (Hook.f. 1890). Potential Value: Medicinal (Oudhia 2012a).

Recorded locality: Chittagong (lnc; Hook.f. 1890, Prain 1903). CHT (lnc; Heinig 1925). No report of collection after Heinig (1925) by its synonym Doritis wightii (Reichb.f.) Benth. and Hook.f.

Golbal distribution: Bangladesh, Cambodia, China, India, Indonesia, Laos, Malaysia, Myanmar, Nepal, Philippines, Sri Lanka, Thailand and Vietnam.

Herbarium specimens: No herbarium specimens are available in DACB, DUSH, HCU, BFRIH and BCSIRH.

42. Podochilus khasianus Hook.f., Fl. Brit. India 6: 81 (1890).

Podochilus chinensis Schltr. (1924). Bangla name: Not known. Habitat: Epiphytic or Lithophytic. Fl.Fr.: March - May (Pearce and Cribb 2002). Potential Value: Horticultural.

Recorded locality: Sylhet (lnc). No report of collection Hook.f. (1890).

Golbal distribution: Bangladesh, Bhutan, China, India and Vietnam.

Herbarium specimens: Sylhet: F. De Silva, Wall. Cat. 7335B (K-W). But no herbarium specimens available in DACB, DUSH, HCU, BFRIH and BCSIRH.

43. Pteroceras teres (Blume) Holttum in Kew Bull. 14: 217 (1960).

Aerides suaveolens Roxb. (1832); Dendrocolla teres Blume (1825); Ornitharium striatulum Lindl. \& Paxton (1851); Pteroceras radicans Hassk. (1842); Sarcochilus suaveolens (Roxb.) Hook.f. (1890). Bangla name: Not known. Habitat: Epiphytic. Fl.-Fr.: June (Pearce and Cribb 2002). Potential Value: Horticultural.

Recorded locality: Chittagong (lnc). No report of collection after Hook.f. (1890).

Golbal distribution: Bangladesh, Borneo, Cambodia, eastern Himalayas, India, Java, Laos, Lesser Sunda Islands, Moluccas, Myanmar, Nepal, Thailand, the Philippines, Sulawesi, Sumatra and Vietnam.

Herbarium specimens: No herbarium specimens are available in DACB, DUSH, HCU, BFRIH and BCSIRH.

44. Schoenorchis gemmata (Lindl.) Smith, Nat. Tijdschr. Ned.-Indie. 72: 100 (1912).

Cleisostoma gemmatum (Lind.) King \& Pantl. (1899); C. dichroanthum Gagnep. (19510; C.leisostoma evrardii Gagnep. (1932); C. gemmatum (Lindl.) King and Pantl. (1899); Gastrochilus gemmatus (Lindl.) Kuntze (18910; Saccolabium hainanense Rolfe (1894); S. geminatum Lindl. (1838); S. spatulatum Rolfe ex Downie (1925). Bangla name: Not known. Habitat: Epiphytic. Fl.-Fr.: May - June (Pearce and Cribb 2002). Potential Value: Horticultural.

Recorded locality: Sylhet (lnc). No report of collection Hook.f. (1890).

Golbal distribution: Bangladesh, Bhutan, Cambodia, China, India, Laos, Myanmar, Nepal, Thailand and Vietnam.

Herbarium specimens: No herbarium specimens are available in DACB, DUSH, HCU, BFRIH and BCSIRH.

45. Spathoglottis pubescens Lindl., Gen. Sp. Orchid. Pl.: 120 (1831).

Epipactis graminifolia Roxb. (1832); Pogonia graminifolia (Roxb.) Voigt (1845); Spathoglottis bensonii Hook.f. (1890); S. fortunei Lindl. (1845); S. khasyana Griff. (1851); S. parvifolia Lindl. (1845); S. pubescens var. parvifolia (Lindl.) Hook.f. (1890). Bangla name: Not known. Habitat: Terrestrial. Fl.Fr.: June - September (Huda 2008). Potential Value: Horticultural. 
Recorded locality: Sylhet (lnc). No report of collection Lindley (1830 - 1840).

Golbal distribution: Bangladesh, Bhutan, Cambodia, China, Hong Kong, India, Laos, Myanmar, Thailand and Vietnam.

Herbarium specimens: East Bengal: 14.10.1835, Griffith $5194(\mathrm{~K})$. But no herbarium specimens are available in DACB, DUSH, HCU, BFRIH and BCSIRH.

46. Tainia latifolia (Lindl.) Reichb.f., Bonplandia 5: 54 (1857).

Ania latifolia Lindl. (1831); Cymbidium fuscescens Griff. (1851); Mitopetalum latifolium (Lindl.) Blume (1856); Tainia angulata (Reichb.f.) Benth. ex Kraenzl. (1911); T. cordata Hook.f. (1890); T. hastata (Lindl.) Hook.f. (1890); T. hologlossa (E.C. Parish and Rchb.f.) Benth. ex Hook.f. (1890); T. khasiana Hook.f. (1890). Bangla name: Not known. Habitat: Terrestrial. Fl: March (Pearce and Cribb 2002). Potential Value: Horticultural.

Recorded locality: Sylhet (lnc). No report of collection Hook.f. (1890).

Golbal distribution: Bangladesh, Bhutan, Cambodia, China, India, Java, Laos, Myanmar, Thailand and Vietnam.

Herbarium specimens: Sylhet: Wallich, Wall. Cat. 3741 (K-W, K). But no herbarium specimens are available in DACB, DUSH, HCU, BFRIH and BCSIRH.

47. Thecostele alata (Roxb.) Parish and Reichb. f. in Trans. Linn. Soc. 30: 135 and 144, t. 29 (1874). Cymbidium alatum Roxb. (1832); C. annamense Gagnep. (1950); C. wrayi Hook.f. (1890); Thecostele maculosa Ridl. (1893); T. zollingeri Reichb. f. (1857). Bangla name: Not known. Habitat: Epiphytic. Fl.: July (Huda 2000). Potential Value: Horticultural.

Recorded locality: Chittagong (lnc). No report of collection after Hook.f. (1890) by its synonym Thecostele zollingeri Reichb. f. (1857).

Golbal distribution: Bangladesh, Borneo, Java, Laos, Malayasia, Myanamar, Sumatra, Thailand, the Philippines and Vietnam.

Herbarium specimens: No herbarium specimens are available in DACB, DUSH, HCU, BFRIH and BCSIRH.

48. Trias oblonga Lindl., Gen. Sp. Orch. Pl.: 60 (1830).

Bulbophyllum burkillii Gage (1906); B. oblongum (Lindl.) Reichb.f (1864); B. moulmiense Reichb.f (1861); Dendrobium tripterum Wall. (1890); Trias ovata Lindl. (1830). Bangla name: Not known. Habitat: Epiphytic. Fl.-Fr.: Spring (IOSPE PHOTOS). Potential Value: Horticultural.

Recorded locality: Sundarbans. No report of collection Prain (1903).

Golbal distribution: Bangladesh, India, Myanmar and Thailand.

Herbarium specimens: No herbarium specimens are available in DACB, DUSH, HCU, BFRIH and BCSIRH.

49. Tropidia angulosa (Lindl.) Blume, Coll. Orchid: 122 (1859).

Cnemidia angulosa Lindl. (1833); C. semilibera Lindl. (1833); Decaisnea angulosa (Lindl.) Wall. (1828-49) ined.; Govindooia nervosa Wight (1853); Tropidia govindovii Blume (1859); T. semilibera (Lind1.) Blume (1859). Bangla name: Angupida. Habitat: Terrestrial. Fl.-Fr.: August - November (Pearce and Cribb 2002). Potential Value: Medicinal (Chopra et al. 1969).

Recorded locality: Sylhet (lnc; Hook.f. 1890), Khulna (lnc). No report of collection after Khalil from Khulna in 1941.

Golbal distribution: Bangladesh, Bhutan, China, India, Indonesia, Japan, Java, Malaysia, Myanmar, Sumatra, Thailand, the Philippines and Vietnam. 
Herbarium specimens: Khulna: 10.06.1941, Khalil s.n. (DUSH) but no herbarium specimens are available in DACB, HCU, BFRIH and BCSIRH.

50. Uncifera acuminata Lindl., J. Proc. Linn. Soc., Bot. 3: 40 (1859).

Saccolabium acuminatum (Lindl.) Hook.f. (1890). Bangla name: Not known. Habitat: Epiphytic. Fl.Fr.: July - September (Pearce and Cribb 2002). Potential Value: Horticultural.

Recorded locality: CHT (lnc). No report of collection after Lister (1876).

Golbal distribution: Bangladesh, Bhutan, China, India and Nepal.

Herbarium specimens: No herbarium specimens are available in DACB, DUSH,HCU, BFRIH and BCSIRH.

51. Vanda cristata Lindl., Gen. Sp. Orch. Pl.: 216 (1833).

Aerides cristata (Lindl.) Wall. ex Hook.f. (1890); Vanda striata Reichb.f. (1868). Bangla name: Not known. Habitat: Epiphytic. Fl.: May (Pearce and Cribb 2002). Potential Value: Medicinal (Behera et al. 2013).

Recorded locality: Sylhet (lnc). No report of collection after Hook.f. (1890).

Golbal distribution: Bangladesh, Bhutan, China, India, Nepal and Vietnam.

Herbarium specimens: No herbarium specimens are available in DACB, DUSH, HCU, BFRIH and BCSIRH.

52. Vanilla aphylla Blume, Bijdr.: 422 (1825).

Vanilla calopogon Reichb.f. (1878); V. parishii Reichb.f. (1878). Bangla name: Vanilla Orchid. Habitat: Hemi-epiphytic or terrestrial. Fl.-Fr.: Not on record. Potential Value: Horticultural.

Recorded locality: Chittagong (lnc; Prain 1903), HT (Barkal; Heinig 1925). No report after Heinig (1925).

Golbal distribution: Bangladesh, India, Java, Laos, penninsular Malaysia, Myanmar, Philippines, Thailand and Vietnam.

Herbarium specimens: No herbarium specimens are available in DACB, DUSH, HCU, BFRIH and BCSIRH.

53. Vrydagzynea albida (Blume) Blume, Coll. Orchid: 71 (1858).

Hetaeria albida Blume (1825); Physurus viridiflorus Lindl. (1857); Vrydagzynea viridiflora (Lindl.) Hook.f. (1890); V. viridifolia Hook. f. (1890). Bangla name: Not known. Habitat: Terrestrial. Fl.-Fr.: September - November (Pearce and Cribb 2002). Potential Value: Horticultural.

Recorded locality: Lakshmipur (lnc). No report of collection after Hook.f. (1890) by its synonym Vrydagzynea viridifolia Hook. f. (1890)

Golbal distribution: Bangladesh, Borneo, India, Java, Malayisa, New Guinea, Sumatra, Thailand, the Philippines and Vietnam.

Herbarium specimens: No herbarium specimens are available in DACB, DUSH, HCU, BFRIH and BCSIRH.

54. Zeuxine affinis (Lindl.) Benth. ex Hook.f., Fl. Brit. India 6: 108 (1890).

Adenostylis arisanensis (Hayata) Hayata (1915); Monochilus affinis Lindl. (1840); Zeuxine arisanensis Hayata (1914); Z. sutepensis Rolfe ex Downie (1925); Z. taiwaniana S.S.Ying (1987). Bangla name: Not known. Habitat: Terrestrial. Fl.-Fr.: February April (Lang et al. 1999). Potential Value: Medicinal (Oudhia 2012).

Recorded locality: Rangamati (lnc) (Barbhuiya and Gogoi 2010). No collection after Wenger's collection in 1939).

Golbal distribution: Bangladesh, Bhutan, China, India, Laos, Malaysia, Myanmar, Nepal, Thailand and Vietnam.

Herbarium specimens: Rangamati: May, 1939, W.J.L. Wenger 23481 (ASSAM) and No herbarium specimens are available in DACB, DUSH, HCU, BFRIH and BCSIRH. 


\section{REFERENCES}

Abraham, A. and P. Vatsala. 1981. Introduction to Orchids. Tropical Botanic Garden and Research Institute. Trivandrum, India. $533 \mathrm{pp}$.

Ahmed, M. and M. K. Pasha. 1993. A taxonomic account of Thrixspermum Lour. (Orchidaceae) from Bangladesh. J. Asiat. Soc. Bangladesh Sci. 19(1): 35-42.

Ahmed, M. and M. K. Pasha. 1994. A taxonomic account of Hetaeria Blume (Orchidaceae) from Bangladesh. Chittagong Univ. Stud. part II. Sci. 18(2): 179-182.

Ahmed, M. and M. K. Pasha. 1998. A taxonomic account of Luisia Gaud. (Orchidaceae) from Bangladesh. J. Bom. Nat. Hist. Soc. 95: 301-306.

Ahmed, M. and M. K. Pasha. 1998a. Gastrochilus inconspicum (Hook.f.) Siedenf. (Orchidaceae)-A new angiospermic record for Bangladesh. Bangladesh J. Bot. 27(2): 61-69.

Ahmed, M., M. K. Pasha and M. A. Aziz Khan. 1989. Agrostophyllum khasianum Griff. (Orchidaceae) - A new record for Bangladesh. Bangladesh J. Bot. 18(1): 101-104.

Ahmed, M., M. K. Pasha and M. A. Aziz Khan. 1989a. Phalaenopsis cornu-cervi (Breda) Par. and Reichb.f. (Orchidaceae) - A new record for Bangladesh. Bangladesh J. Bot. 18(1): 105-108.

Ahmed, M., M. K. Pasha and M. A. Aziz Khan. 1989b. Eria flava Lindl. (Orchidaceae)-A new record for Bangladesh. Bangladesh J. Bot. 18(2): 223-226.

Ahmed, M., M. K. Pasha and M. A. Aziz Khan. 1989c. A taxonomic account of Aerides Lour. (Orchidaceae) from Bangladesh. Bangladesh J. Bot. 18(2): 147-155.

Ahmed, M., M. K. Pasha and M. A. Aziz Khan. 1991. Diploprora championi (Lindl.) Hook.f. (Orchidaceae) - A new record for Bangladesh. Bangladesh J. Bot. 20(2): 101-103.

Ahmed, M., M. K. Pasha and M. A. Aziz Khan. 1993. A taxonomic account of Sarcanthus Lindl. (Orchidaceae) from Bangladesh. J. Eco. Tax. Bot. 17: 487-491.

Ahmed, M., M. K. Pasha and M. A. Aziz Khan 1994. A taxonomic account of Bulbophyllum Thou. (Orchidaceae) from Bangladesh. J. Bom. Nat. Hist. Soc. 91(1): 78-85.

Arefin, M. K., M. M. Rahman, M. Z. Uddin and M. A. Hassan, 2011. Angiospermic flora of Satchari National Park, Habiganj, Bangladesh. Bangladesh J. Plant Taxon. 18(2): 3117-3140.

Barbhuiya, H. A. and R. Gogoi. 2010. Plant collection from Bangladesh in the Herbarium at Shillong (ASSAM), India. Bangladesh J. Plant Taxon. 17(2): 141-165.

Behera, D., C. C. Rath and U. Mohapatra. 2013. Medicinal Orchids in India and their Conservation: A Review. Floricult. Ornamen. Biotech. 7(1): 53-59.

Bhattacharjee, B. and B. K. Dutta. 2010. Flowering Phenology on the Orchids of Barak Valley, Assam, India. Assam Univ. J. Sci. Tech. Biol. Environ. Sci. 6(I): 66-70.

Biswajit, C. 1987. Taxonomic studies of Orchids from Chittagong. M. Sc. Thesis. Department of Botany, University of Chittagong, Bangladesh. 128 pp.

Bose, T. K., S. K. Bhattacharjee, P. Das and U. C. Basak 1999. Orchids of India. Naya Prakash, Calcutta, India. $487 \mathrm{pp}$.

Cai, J., X. Lui, K. Vanneste, S. Proost, W. C. Tsai, K. W. Lui 2015. The genome sequence of the orchid Phalaenopsis equistris. Nature Genetics. 47: 65-72. 
Chopra, R. N., I. C. Chopra and B. S. Varma. 1969. Supliment of Glossary of Indian Medicinal Plants. Council for Scientific and Industrial Research, New Delhi, India.

Datta, R. M. and J. N. Mitra 1953. Common plants in and around Dacca. Bull. Bot. Soc. Beng. 7(1 and 2): 1-10.

Deb, C. R., M. S. Deb, N. S. Jamir and T. Imchen. 2009. Orchids in indigenous system of medicine in Nagaland, India. Pleione. 3(2): 209-221.

Gogoi, K., R. Das, S. Rai and R.Yonzone. 2014. Orchids in Assam, India: The Genus DENDROBIUM Swartz. MIOS J. 15(8): 5-24.

Halim, M and M. S. Khan. 1991. Eulophia mackinnoni Duthie (Orchidaceae) - A new record for Bangladesh. Bangladesh J. Bot. 20(2): 231-233.

Heinig, R. L. 1925. List of Plants of the Chittagong Collectorate and Hill Tracts. The Bengal Government Branch Press, Darjeeling, India. 84 pp.

Hooker, J. D. 1885-1890. The Flora of British India. L. Reeve \& Co. Ltd., Kent, England. 5: 667-910.

Hooker, J. D. 1890-1894. The Flora of British India. L. Reeve \& Co. Ltd., Kent, England. 6: 1-198.

Hossain, M. M. 2011. Therapeutic orchids: Traditional uses and recent advances-An overview. Fitoterapia. 82: 102-140.

Huda, M. K. 2000. Diversity, Ecology, Reproductive biology and Conservation of Orchids of South East Bangladesh. Ph. D. Thesis. Department of Ecology and Soil Science. Aberdeen University, UK. 266 pp.

Huda, M. K. 2007. An updated enumeration of the family Orchidaceae from Bangladesh. J. Orchid Soc. India. 21: 35-49.

Huda, M. K. 2008. Orchidaceae. In: Z. U. Ahmed, M. A. Hassan, Z. N. T. Begum, M. Khondker, S. M. H. Kabir, M. Ahmad, A. T. A. Ahmed, A. K. A. Rahman and E. U. Haque. (eds.). Encyclopedia of Flora and Fauna of Bangladesh. Asiatic Society of Bangladesh, Dhaka. 12: 1-149.

Huda, M. K. and M. A. Rahman 1999. Some rare ornamental epiphytic orchids of potential horticultarul value. Biodiversity Newsletter Bangladesh. 3(1): 2.

Huda, M. K., M. A. Rahman and C. C. Wilcock. 1999. A preliminary checklist of orchid taxa occurring in Bangladesh. Bangladesh J. Plant Taxon. 6(1): 69-85.

Huda, M. K., M. A. Rahman and C. C. Wilcock. 2001. Notes on the Orchidaceae of Bangladesh 1: some new records. Bangladesh J. Plant Taxon. 8(2): 9-17.

Huq, A. M. and M. S. Khan. 1984. A preliminary taxonomic report on the angiospermic flora of Moheskhali Island-1. Dhaka. Univ. Stud. Part B. 32(2): 19-31.

Islam, M. M., M. K. Huda and M. Halim 2016. An Enumeration to the Orchids and their conservation status in Greater Sylhet, Bangladesh. Bangladesh J. Plant Taxon. 23(1): 13-25.

Islam, M. R., M. Z. Uddin and M. A. Hassan. 2009. An assessment of the angiospermic flora of Ramgarh upazila of Khagrachari district, Bangladesh. Bangladesh J. Plant Taxon. 16 (2): 115-140.

IUCN - The World Conservation Union, 2001. IUCN Red List Categories and Criteria. Version 3.1. IUCN, Gland, Swiitzerland and Canbridge, U. K. 
Jayaweera, D. M. A. 1981. Orchidaceae. In: M. D. Dassanyaka and W. D. Fosbergh (eds.). A Revised Handbook to Flora of Ceylon. Amerind Publishing Co. Ltd. New Delhi, India. 2: 1-386.

Joseph, J. 1987. Orchids of Nilgiris. Botanical Survey of India. Govt. Press of India. 186 pp.

Khanam, M., M. Z. Uddin, M. S. Khan and M. A. Hassan. 2001. Our present knowledge on the terrestrial orchidaceous taxa from Bangladesh. Bangladesh J. Plant Taxon. 8(2): 35-49.

Khan, M. S. and M. Hali. 1987. Bulbophyllum lilacinum Redley-A new angiospermic record for Bangladesh. Bangladesh J. Bot. 16(2): 203-205.

Khan, M. S. and M. Halim 1991. Eulophia nuda Lindl.-A new angiospermic record for Bangladesh. Bangladesh J. Bot. 20(1): 81-83.

Khan, M. S. and M. A. Huq. 2001. The vascular flora of Chunati wildlife sanctuary in south Chittagong. Bangladesh J. Plant Taxon. 8(1): 47-64.

Khan, M. S., M. M. Rahman, M. A. Huq, M. M. K. Mia and M. A. Hassan. 1994. Assessment of Biodiversity of Teknaf Game Reserve in Bangladesh focusing on economically and ecologically important plant species. Bangladesh J. Plant Taxon. 1(1): 21-33.

Ken, F. 2014. The Useful Tropical Plants Database, web interface by Ajna Fern with help from Richard Morris.

Kirtikar K. R. and B. D. Basu. 1981. An ICS Indian medicinal plants. 2nd ed. International Book Distributor, Dehradun, India., pp. 2399-2415.

Kurz, W. S. 1877. Forest Flora of British Burma. Bishen Singh Mahendra Pal Singh, Dehra Dun, India. 2: $1-613$.

Lang, K. Y., S. C. Chen, Y. B. Luo and G. H. Zhu 1999. Flora Reipublicae Popularis Sinicae. Science press, Beijing, China. 17: 1-499.

Chen, S. C., Z. H. Tsi, K. Y. Lang and G. H. Zhu. 1999. Flora Reipublicae Popularis Sinicae. Science press, Beijing, China. 18: 1-412.

Tsi, Z. H, S. C. Chen, Y. B. Luo and G. H. Zhu. 1999. Flora Reipublicae Popularis Sinicae. Science press, Beijing, China. 19: 1-437.

Lindley, J. 1830-1840. The Genera and Species of Orchidaceous Plants. Bishen Singh Mahendra Pal Singh, Dehra Dun, India. 565 pp.

Momen, R. U., M. K. Hossain, S. M. S. Huda and B. M. Khan. 2006. Assessment of plant diversity in Chittagong University campus. J. Forest Environ. 4: 27-52.

Moyeen, M. 1982. Taxonomic studies in the family Orchidaceae from Bangladesh. M. Sc. Thesis. Department of Botany, University of Chittagong, Bangladsh. 202 pp.

Nongdam, P. 2014. Ethno-medicinal Uses of some Orchids of Nagaland, North-east India. Res. J. Med. Plant. 8: 126-139.

Oudhia, P. 2012. Interaction with the Indian Traditional Healers on Forest herb for Eternal Stamina and Vigor, Research and Media Network. Bringing people together to improve communication of research findings. Jungle Diaries, Part-15. 
Oudhia, P. 2012a. Medicinal Plants of India with reference to Healing Flora of Andhra Pradesh, Assam, Karnataka, Kerala, Chhattisgarh, Gujarat, Jharkhand, Madhya Pradesh, Maharashtra, Meghalaya, Sikkim, Arunachal Prade. www.pinterest.com.

Pant, B. 2013. Medicinal orchids and their uses: Tissue culture a potential alternative for conservation Afr. J. Plant Sci. 7(10): 448-467.

Pant, B., M. R. Paudel, M. B. Chand and S. H. Wagner. 2016. A Treasure Trove of Orchids in Central Nepal. Department of Botany, Tribhuvan University, Kathmandu, Nepal.

Patil, M. C. and R. T. Mahajan, 2013. Ethnobotanical potential of Eulophia species for their Possible biological activity. Int. J. Pharm. Sci. Rev. Res. 21(2): 297-307.

Pearce, N. R. and P. J. Cribb. 2002. The Orchids of Bhutan. Including a record of Plants from Sikkim and Darjeeling. Vol. 3(3). Royal Botanic Garden, Edinburgh and Royal Government of Bhutan. $643 \mathrm{pp}$.

Prain, D. 1903a. Bengal Plants. Botanical Survey of India, Bishen Singh Mahendra Pal Singh, Dehra Dun, India. 2: 750-777.

Rahman, M. A. (ed.). 2013. Red Data Book of Flowering plants of Bangladesh. Vol. 1. Chittagong, Bangladesh. 272 pp.

Rahman, M. A. and I. Jahan. 2016. An enumeration of the medicinal species of the Fabaceae presumably Extinct from the flora of Bangladesh. J. biodivers. conserv. bioresour. Manag. 2(2): 6982.

Rahman, M. A. and M. K. Huda. 1998. Some threatened and endangered orchids of Bangladesh and their conservation. Biodiversity Newsletter Bangladesh. 2(1): 3-4.

Rahman, M. A. and M. K. Huda. 2001. Notes on the occurrence of Brachycorythis helferi (Reichb.f.) Summerh. - in south-east Bangladesh. Biodiversity Newsletter Bangladesh. 5(1 and 2): 3.

Rahman, M. A. and S. B. Uddin. 1997. Assessment of plant diversity of Sitakunda in Chittagong. Bangladesh J. Plant Taxon. 4(1): 17-36.

Rahman, M. A., M. K. Huda and M. E. Rashid. 2017. Orchid species diversity in Bangladesh and their revised nomenclatural updates. Biod. Bull. Bangladesh. 10: 1-63.

Rahman, M. A., M. N. Uddin, M. E. Rashid, M. N. Uddin and M. M. Islam. 2012. Floristic Diversity in Rampahar reserve forest of Kaptai, Rangamati. Biod. Bull. Bangladesh. 6: 1-31.

Rashid, S. H. and M. M. K. Mia. 2001. Angiospermic flora of Madhupur National Park, Tangail, Bangladesh. Bangladesh J. Plant Taxon. 8(2): 63-82.

Ratnaweera, P. B., D. E. Williams, E. D. Silva, R. L. C. Wijesundera, D. S. Dalisay and R. J. Andersen. 2014. Helvolic acid, an antibacterial nortriterpenoid from a fungal endophyte, Xylaria sp. of orchid Anoectochilus setaceus endemic to Sri Lanka. Int. J. Fungal Biol. 5(1): 23-28.

Roxburgh, W. 1814. Hortus Bengalensis (nom. nud.). Boerhaave Press, Leiden (Holland). 105 pp.

Roxburgh, W. 1832. Flora Indica. In: W. Carey (ed.). Description of Indian Plants. Vol. 3. Mission Press, Serampore, Calcutta, India. 875 pp.

San, M. M., N. M. Aung, H. S. Soe and Y. M. M. Kyaw. 2015. Study on Distribution and Medicinal Values of Wild Orchids in Matu Pe Township, Southern Chin State. Leaflet No. 30/2015, The 
Republic of the Union of Myanmar Ministry of Environmental Conservation and Forestry Forest Department.

Seidenfaden, G. 1978a. Orchid genera in Thailand 6. Neottoideae. Kansk Bot. Ark. 32: 1-195.

Seidenfaden, G. 1978b. Orchid genera in Thailand 7. Oberonia Lindl. and Malaxis Sol ex Sw. Kansk Bot. Ark. 33(1): 1-94.

Seidenfaden, G. 1985. Orchid genera in Thailand 12. Dendrobium Sw. Opera Bot. 83: 26-57.

Seidenfaden, G. 1988. Orchid genera in Thailand XIII. Thirty three Epidendroid genera. Opera Bot. 89: $1-216$.

Sinclair, J. 1956. The Flora of Cox's Bazar, East Pakistan. Bull. Bot. Soc.Beng. 9(2): 1-116.

Tutul, E., M. Z. Uddin, M. O. Rahman and M. A. Hassan. 2009. Angiospermic flora of Runctia Sal Forest, Bangladesh-1. Liliopsida (Monocots). Bangladesh J. Plant Taxon. 16(1): 83-90.

Uddin, M. G., M. A. Rahman and M. E. Rashid. 2013. Plant diversity in Upper Rezu Reserve forest of Ramu, Cox's Bazar. Biod. Bull. Bangladesh. 7: 1-26.

Uddin, S. B. and M. A. Rahman. 1999. Angiospermic flora of Himchari National Park, Cox's Bazar. Bangladesh J. Plant Taxon. 6(1): 31-68.

Uddin, M. Z. and M. A. Hassan. 2004. Flora of Rema-Kalenga Wildlife Sanctuary. IUCN. Bangladesh Country office, Dhaka, Bangladesh., pp. 1-120.

Uddin, M. Z. and M. A. Hassan. 2010. Angiospermic diversity of Lawachara National Park (Bangladesh): A preliminary assessment. Bangladesh J. Plant Taxon. 17(1): 9-22.

Uddin, S. N. and M. A. Hassan. 2012. Angiosperm flora of Rampahar Reserve forest under Rangamati district in Bangladesh. I. Liliopsida (Monocots). Bangladesh J. Plant Taxon. 19(1): 37-44.

Uddin, M. Z., M. A. Hassan and M. S. Khan. 2002. An Annotated Checklist of angiospermic Flora of Rema-Kalenga Wildlife Sanctuary. (Habiganj) in Bangladesh-I. Liliopsida (Monocots). Bangladesh J. Plant Taxon. 9(2): 57-66.

Uddin, S. N., M. S. Khan, M. A. Hassan and M. K. Alam 1998. An Annotated Checklist of angiospermic flora of Sitapahar at Kaptai in Bangladseh. Bangladesh J. Plant Taxon. 5(1): 13-46.

Yonzone, R., D. Lama, R. B. Bhujel and S. Rai. 2012. Taxonomic Assessment on the reported Orchid species of Darjeeling district from flora of Bhutan, the Orchids of Bhutan-A review. Int. J. Pharm. Life Sci. 3(3): 1533-1550.

Wallich, N. 1828-1849. A numerical list of dried specimens of plants in the East Indian Company's Museum, Ined.

Websites: http://www.ecoindia.com/flora/flowers/orchid-plant.html, IOSPE PHOTOS, Kew (World Checklist of Selected Plant Families), the plantlist. 
J. biodivers. conserv. bioresour. manag. 3(1), 2017 